\title{
Coexistence of psoriasis, and alopecia areata with trachyonychia in a pediatric patient with Turner Syndrome
}

\author{
Seval Dogruk Kacar, M.D. Ass. Prof. ${ }^{a}$, Pınar Ozuguz, M.D. Ass. Prof. ${ }^{a}$ and Serap Polat, M.D. ${ }^{a}$
}

\begin{abstract}
Turner syndrome is a genetic disorder characterized by abnormalities of the $X$ chromosome. An association with many autoimmune diseases is described as well as skin diseases such as psoriasis, vitiligo and alopecia areata. Trachyonychia is characterized by irregular opaque appearance on the nail plate of all 20 nails. It may be idiopathic or it can be associated with other dermatological diseases. Herein, we present a 6 years old girl with Turner syndrome who had diagnoses of psoriasis, alopecia areata and trachyonychia and we would like to draw attention to the possible relationship between these diseases. Key words: alopecia areata, psoriasis, trachyonychia, Turner syndrome.
\end{abstract}

http:/ /dx.doi.org/10.5546/aap.2014.eng.e209

\section{INTRODUCTION}

Turner syndrome (TS) is a sporadic genetic disorder characterized by monosomy (45X) or structural defects of the $X$ chromosome. In addition to the typical clinical features, cutaneous findings such as xerosis, numerous melanocytic nevi, halo nevi, keloids and hypoplastic nails can also be observed. ${ }^{1,2}$ The prevalence of autoimmune skin diseases such as psoriasis, vitiligo, lichen planus and alopecia areata is increased in patients with TS. ${ }^{2-4}$ Trachyonychia, also known as twenty-nail dystrophy is characterized by brittle, thin nails, with excessive longitudinal ridging. ${ }^{5}$ This disease may be idiopathic or it can be associated with other dermatological diseases, especially alopecia areata and psoriasis. In its severe form, the roughness of nail can cause opaque sandpaper nails. We present coexistence of inverse psoriasis, alopecia areata and trachyonychia in a pediatric patient with TS.

a. Department of Dermatology and Venereology, Faculty of Medicine, Afyon Kocatepe University, Afyonkarahisar; Turkey.

E-mail Address:

Seval Dogruk Kacar, M.D. Ass. Prof.: sevaldogruk@hotmail.com

Conflict of Interest: none.

Received: 1-28-2014

Accepted: 4-14-2014

\section{Case report}

A 6 years old girl with TS was referred to our outpatient clinic with complaints of patchy hair loss for two months, a two year history of rough nails gradually extanded to both finger and toe nails and scaly lesions in the scalp, ears, eyelids, belly button and genital area present for about 3 years which sometimes waxes and wanes with use of topical corticosteroid creams. She was prenatally diagnosed as TS with 45X karyotype after amniocentesis which was performed after a webbed neck appearence on fetal ultrasonography. Although she had neither edema of hands and feet at birth nor any other sign of this monosomy, the postnatal karyotyping confirmed the diagnosis. On dermatological examination, two alopecic non scaly patches on occipital region of the scalp, irregular, opaque appearance of all finger and toe nails, with longitudinal ridging, koilonychia and cuticular hyperkeratosis were seen. Besides pale erythematous, slightly scaly plaques at occipital scalp, right ear helix, bilateral medial canthus, umbilicus, and anogenital region were observed (Figures 1-3). A trichogram from the margin of alopecic patch showed telogen and dystrophic hairs. Potassium hydroxide standart microscopy from the scales at scalp border and ear for fungal etiology were negative. She had no history of a systemic disease. She was on growth hormone replacement therapy, because of TS. The lesions at scalp and nails, and truncal lesions were evaluated as alopecia areata, trachyonychia and psoriasis, respectively. The thyroid function tests were normal and antinuclear antibodies (ANA) were positive $(1 / 320)$. Thyroid autoantibodies and further autoimmune panel was negative. Topical minoxidil $2 \%$ was applied to alopecic plaques on the scalp, $1 \%$ pimecrolimus cream prescribed for psoriatic plaques and a topical preparation containing $40 \%$ urea combined with topical mometasone furoate for nails were recommended.

\section{DISCUSSION}

TS was defined by the triad of "sexual infantilism, webbed neck, and cubitus valgus". ${ }^{2}$ The 
incidence among liveborn females has been reported as $1 / 2000-1 / 5000 .{ }^{6}$ This disease has many characteristic clinical manifestations such as short stature, webbed neck, cubitus valgus, low posterior hairline and widely spaced nipples. The common dermatologic conditions associated with TS are mainly autoimmune-based diseases, as alopecia areata, psoriasis, vitiligo, halo nevus, Dupuytren contracture. Increased incidence of several autoimmune diseases in patients with TS have also been reported. ${ }^{2,4}$ The reason of this condition is unknown but several theories have been propo-

FIGURE 1. Rough and opaque appearence in all 20 nails

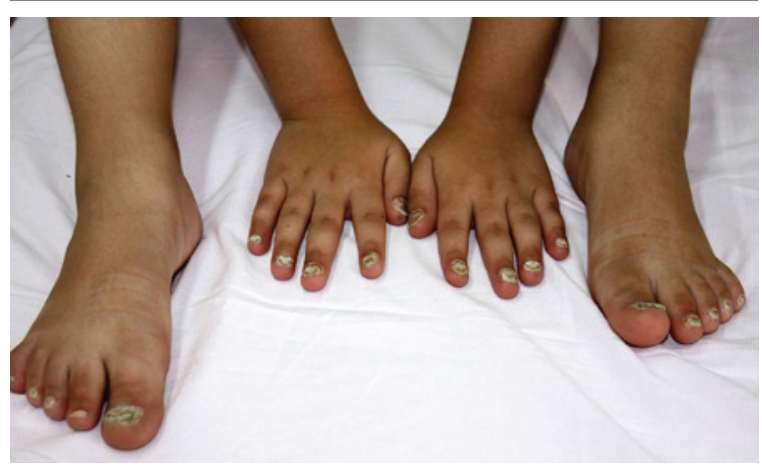

FIGURE 2. Sharp bordered, pale erythematous slight scaly plaques in genitalia

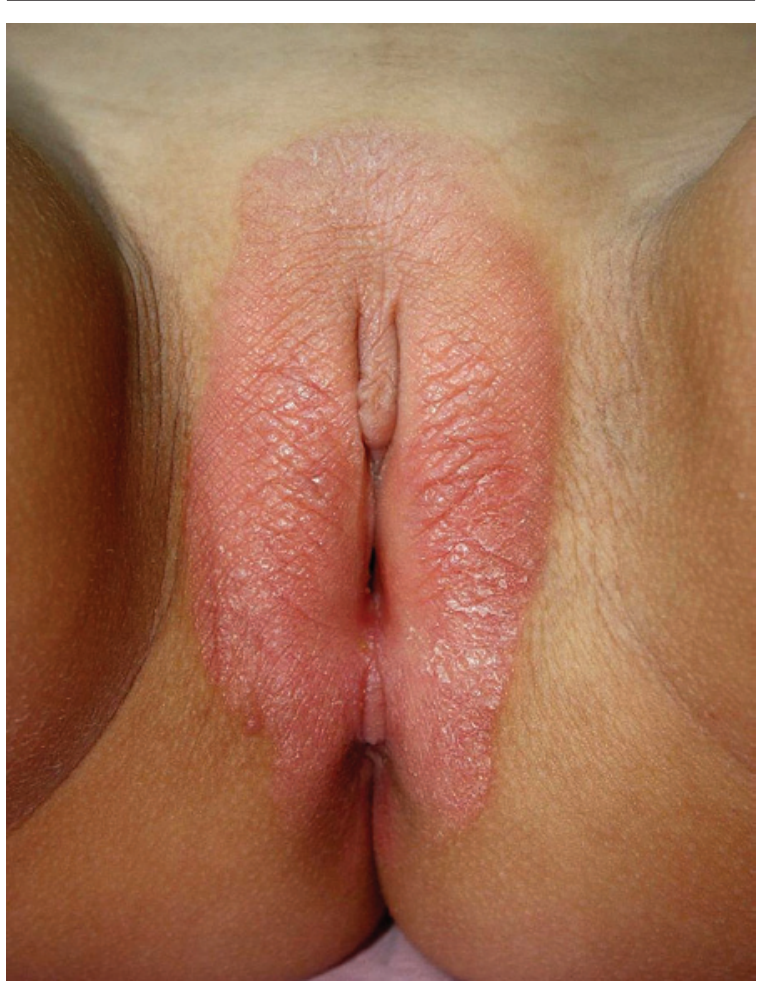

sed. The first theory displays a possible relationship between autoimmunity and nondisjunction, The mainstay is the other nondisjunctional chromosomal disorders like Down syndrome and Klienfelter syndrome also have a higher incidence of autoimmune diseases; and also autoimmune disorders are frequent in families of patients with TS. Thus families with autoimune disorders may be prone to nondisjunctional disorders. ${ }^{7}$ Another hypothesis is that a possible association between autoimmune diseases and $\mathrm{X}$ chromosome genes may exist, as the majority of patients with autoimmune diseases are women. ${ }^{8}$ Haplotypes also seem to be important as haploinsufficiency on the $\mathrm{X}$ chromosome may be responsible for lack of self-protein exposure in the thymus and escape of autoreactive T cells, predisposing autoimmunity. ${ }^{9}$

Furthermore psoriasis is usually improved during pregnancy which was correlated with increased estrogen levels in a study by Murase. ${ }^{10}$ In another study, skin inflammation was related to hormones as a result of variations in natural course of psoriasis, during menstruation. ${ }^{11}$ Estrogen downregulates the production of many chemoki-

FIGURE 3. Alopecic patch in occipital area of scalp

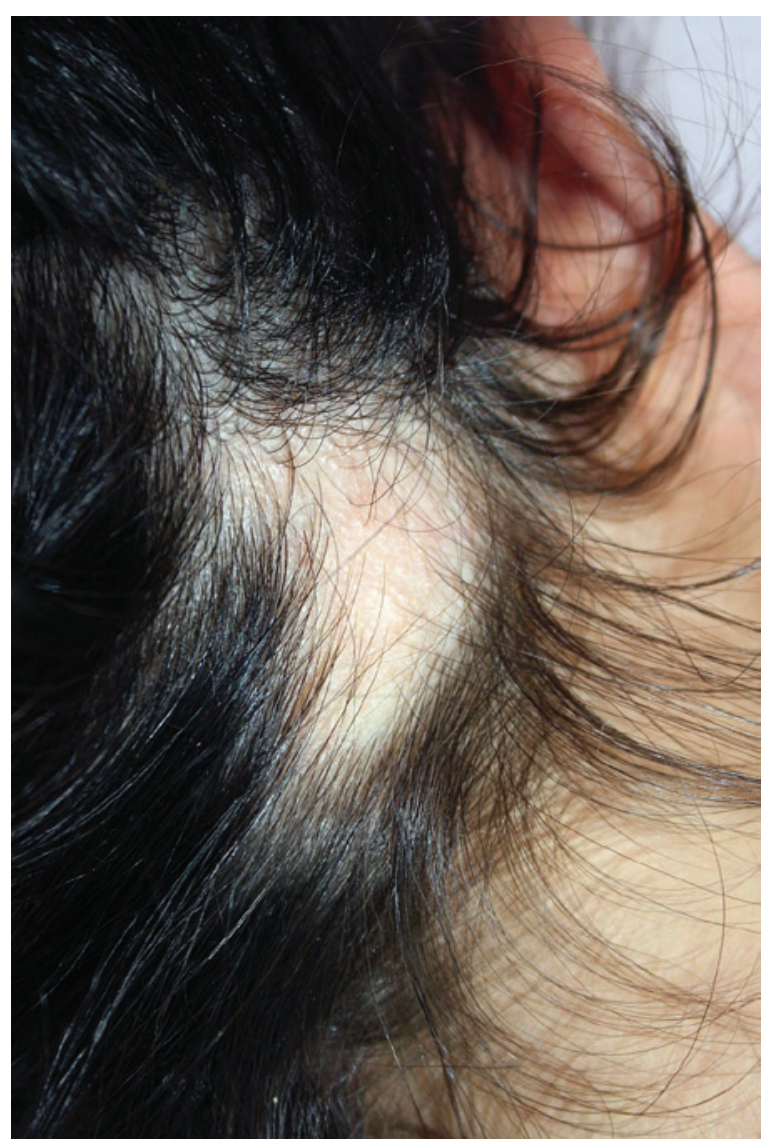


nes that attract neutrophils, T-cell and macrophages, suppress IL-12 production and enhance the antiinflammatory IL-10 production by dendritic cells. As a result, low levels of estrogen may lead to inflammatory changes in skin.

The diagnosis of hair lesions was straight. Tinea capitis which is another common cause of alopecia in children usually presents with scaly annular patches. ${ }^{12}$ It can be excluded by performing a direct microscopy but there was not any desquamation in this case. Trikotillomania was also ruled out with trcihogram which should reveal almost $100 \%$ anagen hair. ${ }^{12}$ The skin lesions were diagnosed clinically, as the typical presentation of inverse psoriasis with sharp edged, erythematous, slightly desquamated plaques in umblicus and labia majora. The scalp and ear lesions were diagnosed as psoriasis after direct microscopic examination to exclude a dermatophyte infection.

Children less than 10 years old with diagnosis of alopecia areata are treated with topical midpotent corticosteroids and topical 5\% minoxidil solution or short contact anthralin. ${ }^{13}$ We preferred topical $2 \%$ minoxidil. In management of psoriasis, topical medications are preferred when the body surface area affected is less than $10 \% .{ }^{14}$ Although topical corticosteroids and vitamin $\mathrm{D}$ analogues are the most common topical drugs prescribed, topical 1\% pimecrolimus is a good alternative especially used in inverse and facial psoriasis as in our case. With disease chronicity and severity, phototherapy and systemic therapies may be given. There are many topical and systemic treatment modalities reported to be successful in trachyonychia, ${ }^{5}$ but spontaneous resolution may occur and we preferred an urea containing preperation to soften and thin nails.

Alopecia areata and psoriasis are both related to autoimmune and immunological mechanisms. Trachyonychia is a clinical entity which may be associated with both diseases. In the setting of psoriasis, patients may have a more thickening of nail plate. ${ }^{5}$ To our knowledge, a case with TS, alopecia areata and psoriasis has been reported, ${ }^{1}$ but this case had no trachyonychia. In our case, trachyonychia was also present.

In conclusion, because of this association, we considered that there may be a genetic association between TS, psoriasis and alopecia areata or a possible common autoimmune pathogenesis.

\section{REFERENCES}

1. Rosina P, Segalla G, Magnanini M, Chieregato C, et al. Turner's syndrome associated with psoriasis and alopecia areata. J Eur Acad Dermatol Venereol 2003;17(1):50-2.

2. Lowenstein EJ, Kim KH, Glick SA. Turner's syndrome in dermatology. J Am Acad Dermatol 2004;50(5):767-76.

3. Lee WS, Yoo MS. Alopecia areata in a patient with Turner's syndrome. Br J Dermatol 1996;135(6):1013.

4. Kurgansky D, Burnett JW. Widespread lichen planus in association with Turner's syndrome and multiple endocrinopathies. Cutis 1994;54(2):108-10.

5. Gordon KA, Vega JM, Tosti A. Trachyonychia: a comprehensive review. Indian J Dermatol Venereol Leprol 2011;77(6):640-5.

6. Hook EB, Warburton D. The distribution of chromosomal genotypes associated with Turner's syndrome: livebirth prevalence rates and evidence for diminished fetal mortality and severity in genotypes associated with structural X abnormalities or mosaicism. Hum Genet 1983;64(1):24-7.

7. Lleo A, Moroni L, Caliari L, et al. Autoimmunity and Turner's syndrome. Autoimmun Rev 2012;11(6-7):A538-43.

8. Torfs CP, van den Berg BJ, Oechsli FW, Christianson RE. Thyroid antibodies as a risk factor for Down syndrome and other trisomies. Am J Hum Genet 1990;47(4):727-34.

9. Bianchi I, Lleo A, Gershwin ME, Invernizzi P. The X chromosome and immune associated genes. J Autoimmun 2012;38(2-3):J187-92.

10. Murase JE, Chan KK, Garite TJ, Cooper DM, et al. Hormonal effect on psoriasis in pregnancy and post partum. Arch Dermatol 2005;141(5):601-6.

11. Kanda N, Watanabe S. Regulatory roles of sex hormones in cutaneous biology and immunology. J Dermatol Sci 2005;38(1):1-7.

12. Mandt N, Vogt A, Blume-Peytavi U. Differential diagnosis of hair loss in children. J Dtsch Dermatol Ges 2004;2(6):399411.

13. Alkhalifah A, Alsantali A, Wang E, McElwee KJ, et al. Alopecia areata update: part II. Treatment. J Am Acad Dermatol 2010;62(2):191-202.

14. Shah KN. Diagnosis and treatment of pediatric psoriasis: current and future. Am J Clin Dermatol 2013;14(3):195-213. 